\title{
El Proyecto Archivo de la Palabra y de la Imagen de Cartagena: el archivo encuentra al archivero
}

The Archivo de la Palabra y de la Imagen de Cartagena Project: the archive meets the archivist

\author{
Raquel Arango Zapata, Rafael Belda González y Juana María Gómez Egea
}

Archivo Municipal de Cartagena. Plaza del General López Pinto s/n. 30201-Cartagena.

palabra1@ayto-cartagena.es; palabra4@ayto-cartagena.es; palabra2@ayto-cartagena.es

\begin{abstract}
Resumen
La presente exposición tiene por finalidad dar a conocer el proyecto Archivo de la Palabra y de la Imagen de Cartagena, que nace de la necesidad de conservar el patrimonio cultural inmaterial de la ciudad y su comarca. Se trata de una información que, de no capturarse y conservarse adecuadamente, corre el peligro de desaparecer, y con ella la memoria oral de comunidades no apoyadas en la escritura. Del proyecto que se muestra en la presente exposición se espera obtener como principales productos una base de conocimiento, compuesta de documentos digitales y sus metadatos de creación, gestión y uso, acerca de la cultura no escrita de la comarca, así como un conjunto de herramientas terciarias.
\end{abstract}

Palabras clave: Archivos. Contexto archivístico. Cultura oral. Metodología etnográfica. Patrimonio cultural material e inmaterial.

\section{Introducción}

La presente exposición tiene por finalidad dar a conocer el proyecto Archivo de la Palabra y de la Imagen de Cartagena. Para ello se explora la noción de la identificación de archivo y archivero, específicamente, en el entorno de la cultura oral; así como la técnica utilizada para explicitar dicha noción, tomando como punto de referencia el método etnográfico.

El desarrollo de proyectos culturales basados en la recogida de la historia tradicional de forma oral nace de la necesidad de conservar el patrimonio tanto material como inmaterial dentro del marco geográfico en el que se genera. En este sentido la Concejalía de Cultura de la ciudad de Cartagena, a través del Archivo Municipal, puso en marcha el citado proyecto en el año 2005, convirtiéndose así en referente obligado de una tradición oral que anida en la identidad de sus diferentes comunidades. Este tipo de trabajos

\begin{abstract}
The aim of the following exposition is to describe the 'Archivo de la Palabra y de la Imagen de Cartagena' project, which emerges as a need to preserve the intangible cultural heritage of the city of Cartagena and its region. If that information is not researched and preserved adequately, there is a risk that it will disappear. That is to say, we can lose a valuable information transmitted orally-and consequently without any written available documents-by our community. Finally, we hope to obtain from the project we have presented on this document a base of knowledge made up of digital documents and their metadata of creation, administration and use about nonwritten culture of our region, and some tertiary tools as well.
\end{abstract}

Keywords: Archives. Archival context. Oral cultures. Ethnographical methodology. Material heritage.

adquieren especial relevancia en los últimos años, a partir de las conclusiones extraídas de la Convención del Patrimonio Cultural Inmaterial promovida por la UNESCO en el año 2003. Dicha Convención reconoce que las comunidades indigenas, las comunidades de práctica, los grupos y, en algunos casos, los individuos, desempeñan un papel importante en la producción, la salvaguardia, el mantenimiento y la recreación del patrimonio cultural inmaterial, contribuyendo con ello a enriquecer la diversidad cultural y la creatividad humana (Unesco 2003).

A efectos de claridad de la exposición, procedemos a definir los términos clave en torno a los cuales se articula el todo del proyecto. Así, descendiendo de lo general a lo específico, abordamos en filigrana las nociones utilizadas de patrimonio cultural inmaterial, de historia oral y de lengua. 
Se entiende por "patrimonio cultural inmaterial" los usos, representaciones, expresiones, conocimientos y técnicas - junto con los instrumentos, objetos, artefactos y espacios culturales que les son inherentes- que las comunidades, los grupos y en algunos casos los individuos reconocen como parte integrante de su patrimonio cultural. Este patrimonio cultural inmaterial, que se transmite de generación en generación, es recreado constantemente por las comunidades y grupos en función de sus interacciones con el entorno, con la naturaleza y con la historia, infundiendo a los individuos un sentimiento de identidad y continuidad (Unesco, 2003).

Thompson (1988) describe la historia oral como "una historia construída en torno a las personas. Introduce la vida en la misma historia y amplia sus horizontes. Reconoce como héroes no sólo a los líderes, sino a la desconocida mayoría de las personas."

Como principal medio de comunicación del ser humano, las lenguas no sólo transmiten mensajes sino que expresan también emociones, intenciones y valores, confirman las relaciones sociales y transmiten expresiones y prácticas culturales y sociales. Ya sea oralmente, por escrito o a través de gestos, las lenguas son el vínculo de la memoria, las tradiciones, el conocimiento y las técnicas. Por lo tanto, la lengua constituye un factor determinante de identidad, y la preservación de las diferentes identidades de las sociedades del mundo refuerza el respeto por la diversidad cultural (Smeets 2006).

Un ejemplo sobre la importancia del estudio de las lenguas es el informe del Estudio de las lenguas indígenas nacionales 2005, elaborado por el Australian Institute of Aboriginal and Torres Strait Islander Studies a petición del gobierno de Australia, donde se expone un análisis de la situación de las lenguas indígenas australianas basado en los nueve factores de la UNESCO para evaluar la vitalidad de una lengua. Entre sus resultados más importantes, el informe afirmaba que solamente 145 de las más de 250 lenguas indígenas conocidas en Australia seguían hablándose realmente. Y de éstas, unas 110 han sido clasificadas como amenazadas gravemente o en estado crítico. Sólo 18 lenguas indígenas se consideran "fuertes" de acuerdo con un factor tan importante como la transmisión intergeneracional (Australian Institute of Aboriginal and Torres Strait Islander Studies 2005). Sirva este ejemplo para justificar la relevancia de la investigación en relación con las culturas orales en trance de extinción, entendiendo por tales, no sólo las indígenas, sino todas aquellas, incluídas las occidentales, que no se adecúan al canon de la globalización.

\section{Aproximación teórica}

La metodología seguida en las primeras fases de desarrollo del proyecto se centra en el método etnográfico, tal y como ha sido propuesto particularmente por Karen F. Gracy (2006) en relación con la etnografía archivística. Gracy argumenta que las raíces de la etnografía

se hunden en el campo de la antropología, donde existe una larga tradición de investigadores que pasan gran parte de su vida viviendo con y estudiando culturas ... Esta expansión de la agenda etnográfica refleja una nueva comprensión de la cultura y de la comunidad ... La etnografía archivística es una forma de pesquisa naturalista que posiciona al investigador dentro de un entorno archivístico para ganar la perspectiva cultural de los responsables de la creación, recogida, cuidado y uso de los documentos.

En la revisión de la revista Archives \& Social Studies: A Journal of Interdisciplinary Research (Delgado Gómez, 2007) se pone de manifiesto la existencia de formas documentales implícitas, y que cada sociedad o grupo conceptualiza el documento y el archivo de diferente manera, dependiendo del modo en que conceptualice nociones como las de evidencia, responsabilidad y memoria; así como que la disciplina archivística, en la actualidad, explora nuevas relaciones con la etnografía.

En el caso de los archivos personales, particularmente de los derivados de comunidades cuyos mecanismos de recuerdo y olvido son esencialmente orales, cabe la posibilidad de considerar que el documento se identifica con la propia memoria de los informantes. En tal caso habría que definir si la memoria es un soporte estable y si existen mecanismos de selección de documentos, valoración, expurgo, descripción etc. La psicología cognitiva y la neurología confirmarían este hecho. Además de en los archivos orales, en los archivos de literatos también puede considerarse que la propia memoria es el archivo. En este caso el archivo y el archivero son indisolubles, son la misma entidad. El archivero (el informante, la persona) ejerce como gestor de los documentos de su propia memoria, siempre y cuando se considere que la memoria es un soporte estable. Esto es mucho más evidente si esa traza de la memoria se queda reflejada en un soporte externo: una fotografía, un texto o un video, una conversación con terceros, etc. En este caso se produce una fijación externa del documento, un esbozo de pluralización del mismo. $Y$ en ese sentido merece explorar la conexión, el nexo entre el archivo y el archivero. Se trataría de la memoria, entendida como otro tipo documental, ajeno a los tipos convencionales. Esto resulta particularmente obvio en cultu- 
ras que carecen de una tradición escrita: culturas primitivas en África, en Asia o en Oceanía, por ejemplo, y que vienen a constituir un setenta o un ochenta por ciento de la humanidad. Este tanto por ciento de la humanidad no tiene una memoria escrita y debe confiar en otro tipo de memoria, y no es el menor la memoria puramente oral. En este caso habría que explorar si el archivo y el archivero no son una y la misma cosa. También habría que explorar si no se deforma ese archivo cuando se traslada a grabaciones sonoras, a grabaciones de video, según el punto de vista positivista occidental.

\section{Objetivos}

Los objetivos que se han tenido en cuenta a la hora de desarrollar el trabajo se apoyan en la anterior argumentación y renuncian a la citada creencia positivista de que, en calidad de archiveros profesionales, podemos salvaguardar la memoria del archivero inconsciente y anónimo, y se concretan en:

- Recopilar datos que permitan crear un archivo oral lo más completo posible, abordando temas que forman parte de lo que se conoce como cultura popular, así como todas aquellas referencias de carácter histórico que se conservan en la memoria de nuestros mayores.

- Recoger materiales de otro tipo en diferentes soportes: fotográficos, documentos escritos, audiovisuales, objetos físicos, etc.

- Proporcionar contexto y detalle, haciendo visible lo que a menudo es invisible.

- Observar conductas e interacciones cotidianas, ya que éstas crean significado y definen valores.

- Capturar la información dentro de una comunidad, de un grupo o de un individuo, entendidos como acumuladores de evidencia documental, y gestionar y poner en uso la misma.

- Participar en la vida y compartir las percepciones de las personas que estudiamos.

- Comprender la realidad de las experiencias de los participantes y cómo las experimentan.

- Aprender más acerca de las personas y prácticas del entorno.

- Enfatizar el contexto, el entorno, la cultura del lugar, así como los marcos de referencia de los participantes.

\section{Secuenciación del proyecto}

La concepción del proyecto determina las cuestiones a documentar, que son de origen tanto etnográfico como histórico. La secuencia para la ejecución del proyecto, a tenor de todo lo dicho, se centra en las siguientes fases:

- Recopilación de datos etnográficos e históricos con el objetivo de poblar una base de datos sobre el pasado, presente y futuro de los modos de vida multiculturales en la ciudad de Cartagena y su comarca. Dicha base de datos se nutre de temáticas diversas, como los denominados ritos de paso, que son fundamentales en el ciclo vital de todo ser humano, y que marcan los momentos más importantes de la existencia. Cada sociedad y cultura los asume desde diferentes perspectivas y tienen como fin defenderse del paso del tiempo y de la crisis que supone la transición entre cada período. De igual modo, se recoge información sobre la persona, su trayectoria y su identidad; la gente, la vecindad y la convivencia en la comunidad; el mundo laboral; la vida de ocio y las fiestas; los ritos y lugares de culto; los espacios domésticos y públicos; la historia en el discurso popular; los procesos de transformación en el espacio y el tiempo, junto con los objetos relacionados con la vida del lugar.

- Elección de las unidades geográficas, rurales y urbanas, y de muestras representativas de individuos capaces de proporcionar información pertinente acerca de las cuestiones a documentar elaboradas durante el diseño de la investigación. Los criterios de selección para la unidad geográfica recogen la existencia e importancia de una marcada identidad local y la tradición de relaciones intercomunales.

- Los criterios de selección para los informantes-claves reflejan la variación de perspectivas de investigación mediante grupos significativos y personas a nivel individual, como miembros destacados dentro de la comunidad; tomando en cuenta variables tales como sexo, edad, origen, nivel social y cultural.

- El trabajo de campo se basa en la investigación participativa y en la entrevista directa con los informantes. En una toma de contacto previa se realiza el análisis de procesos, donde es aconsejable explicar con claridad a los informantes potenciales acerca de qué es lo que se pretende conseguir, cuáles son los objetivos y la finalidad del trabajo en cuestión. Lo más importante es despertar su curiosidad, en un proceso que se va a desarrollar con ellos como principales protagonistas. Con la entrevista etnográfica se recopila información oral 
sobre los acontecimientos pretéritos del lugar: opiniones, motivos y valoraciones de los informantes. Se trata de un proceso comunicativo con diferente grado de implicación del entrevistador (receptivo o activo). La entrevista ha de tener un carácter semi-estructurado con un orden flexible de preguntas y preguntas abiertas que generen textos, narraciones y anécdotas. En cuanto a la tipología de pregunta debemos tener en cuenta las denominadas introductorias ("preguntas rompe-hielo"), las follow-up (señalan seguimiento), preguntas detalladas y específicas, preguntas directas e indirectas, preguntas sugestivas, de sondeo, y el silencio como pregunta.

- Análisis detallado y explotación de la información, mediante métodos cualitativos, y elaboración de herramientas terciarias, como índices y guías temáticas.

\section{Conclusiones}

Del proyecto que se muestra en la presente exposición se espera obtener como principales productos una base de conocimiento compuesta de documentos digitales y sus metadatos de creación, gestión y uso, acerca de la cultura no escrita de la comarca, así como un conjunto de herramientas terciarias. Estos productos debieran servir de apoyo a posteriores investigaciones etnográficas e históricas, y ponerse a disposición de la ciudadanía en general.
La constitución de un archivo oral resulta hoy una empresa obligada por varios motivos:

- Intentar no perder el inmenso patrimonio cultural correspondiente a una sociedad en trance de extinción que anida todavía en la memoria de nuestros mayores

- La experiencia vivida atesorada en el recuerdo de nuestros conciudadanos, debe aportarnos un complemento y un contrapeso del conocimiento basado en textos.

- Una iniciativa semejante situará a la institución que la promueva como ejemplo y referencia para otros proyectos semejantes.

\section{Referencias}

Australian Institute of Aboriginal and Torres Strait Islander Studies (2005): Estudio de las lenguas indígenas nacionales 2005. www.dcita.gov.au/indig/maintenance_indi genous_languages/publications (Consulta: 14-6-2007)

Delgado Gómez, Alejandro (2007). Archives \& Social Studies. // Butlletí de l'Associació d'Arxivers Valencians (en prensa).

Gracy, Karen. F. (2006): Documentar comunidades de práctica: el caso de la etnografía archivística. // Gilliland, Anne; McKemmish, Sue (eds.). Nuevos métodos de investigación en archivística. Cartagena: Ayuntamiento: 3000 Informática, 2006.

Smeets, Rikes (2006). Cuestiones lingüísticas // El Mensajero del Patrimonio Inmaterial. 1 (2006).

Thompson, P. (1988). La voz del pasado: la historia oral. Valencia: Akal,1988.

Unesco (2003). Convención para la salvaguardia del Patrimonio Cultural Inmaterial (2003). París: UNESCO, 2003. 